Supporting Information

\title{
Nanoscale Zeolitic Imidazolate Framework-8 Activator of Canonical MAPK Signaling for Bone Repair
}

Xiaomeng Gao, Yiyuan Xue, Zhou Zhu, Junyu Chen, Yanhua Liu, Xinting Cheng, Xin Zhang, Jian Wang, Xibo Pei* and Qianbing Wan*

State Key Laboratory of Oral Diseases, National Clinical Research Center for Oral Diseases, Department of Prosthodontics, West China Hospital of Stomatology, Sichuan University, Chengdu 610041, Peoples R China

* Corresponding author:

Xibo Pei; e-mail: xbpei@hotmail.com Tel:+86-13541202602 Fax: +86-28-85501450

Qianbing Wan; e-mail: champion@scu.edu.cn Tel:+86-13688339355 Fax: $+86-28-85501450$

Xibo Pei and Qianbing Wan, Sichuan University, No. 14, Section 3, South Peoples Road, Chengdu 610041, China. 


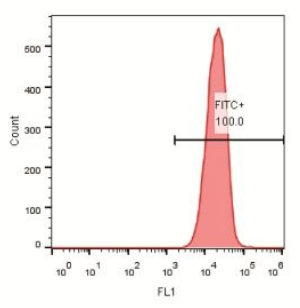

Coso

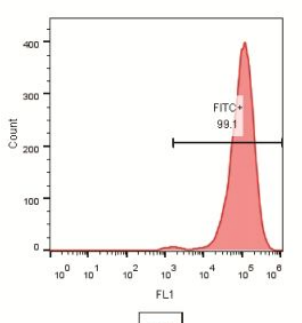

$\mathrm{CO} 29$

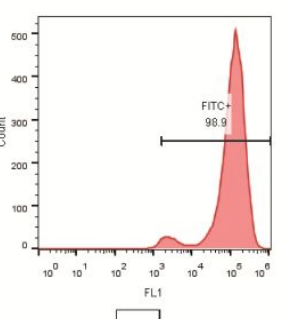

Co44
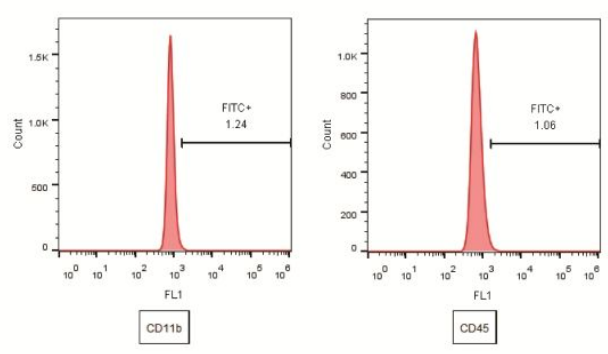

Figure S1. rBMSCs surface markers examined by flow cytometry. 


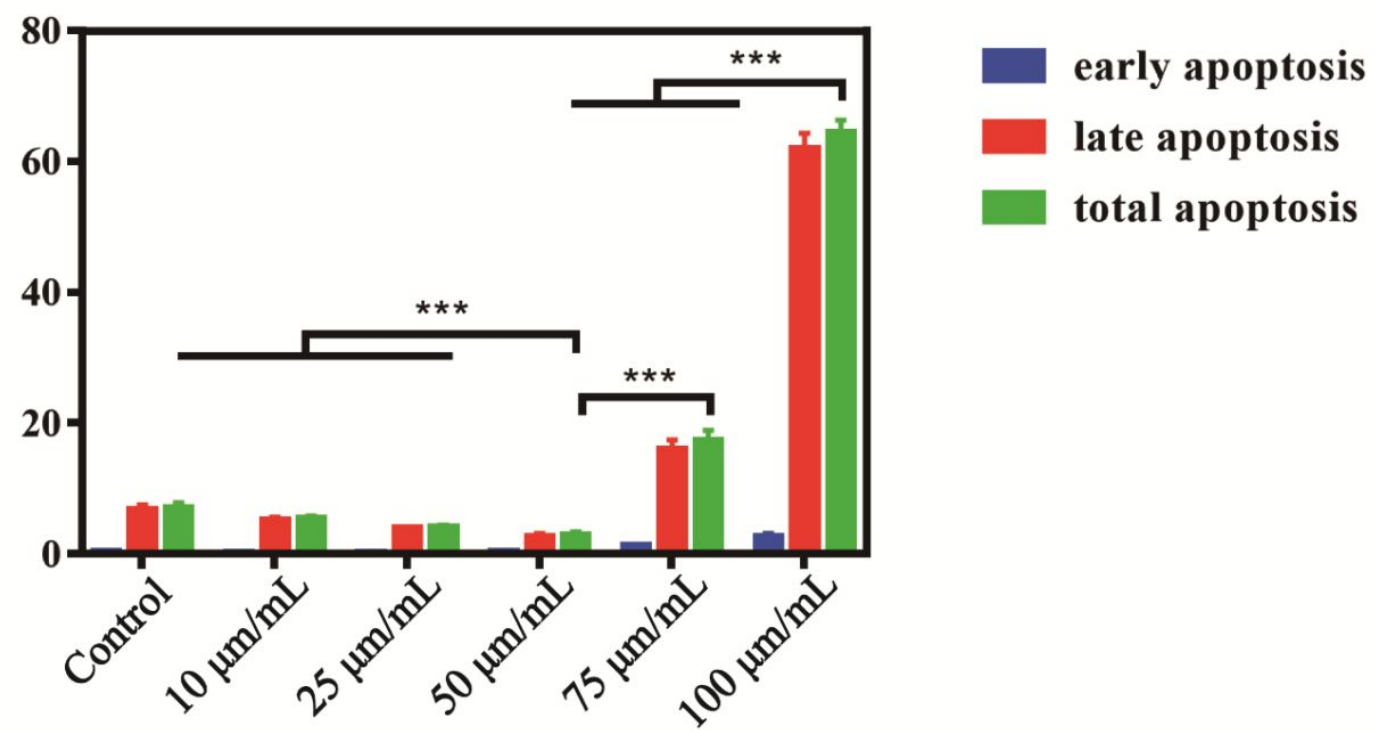

Figure S2. Quantity analysis of apoptosis rBMSCs by nano ZIF-8 at different concentrations. $n=3 .{ }^{*} \mathrm{P}<0.05, * * \mathrm{P}<0.01, * * * \mathrm{P}<0.001$ between each group. 


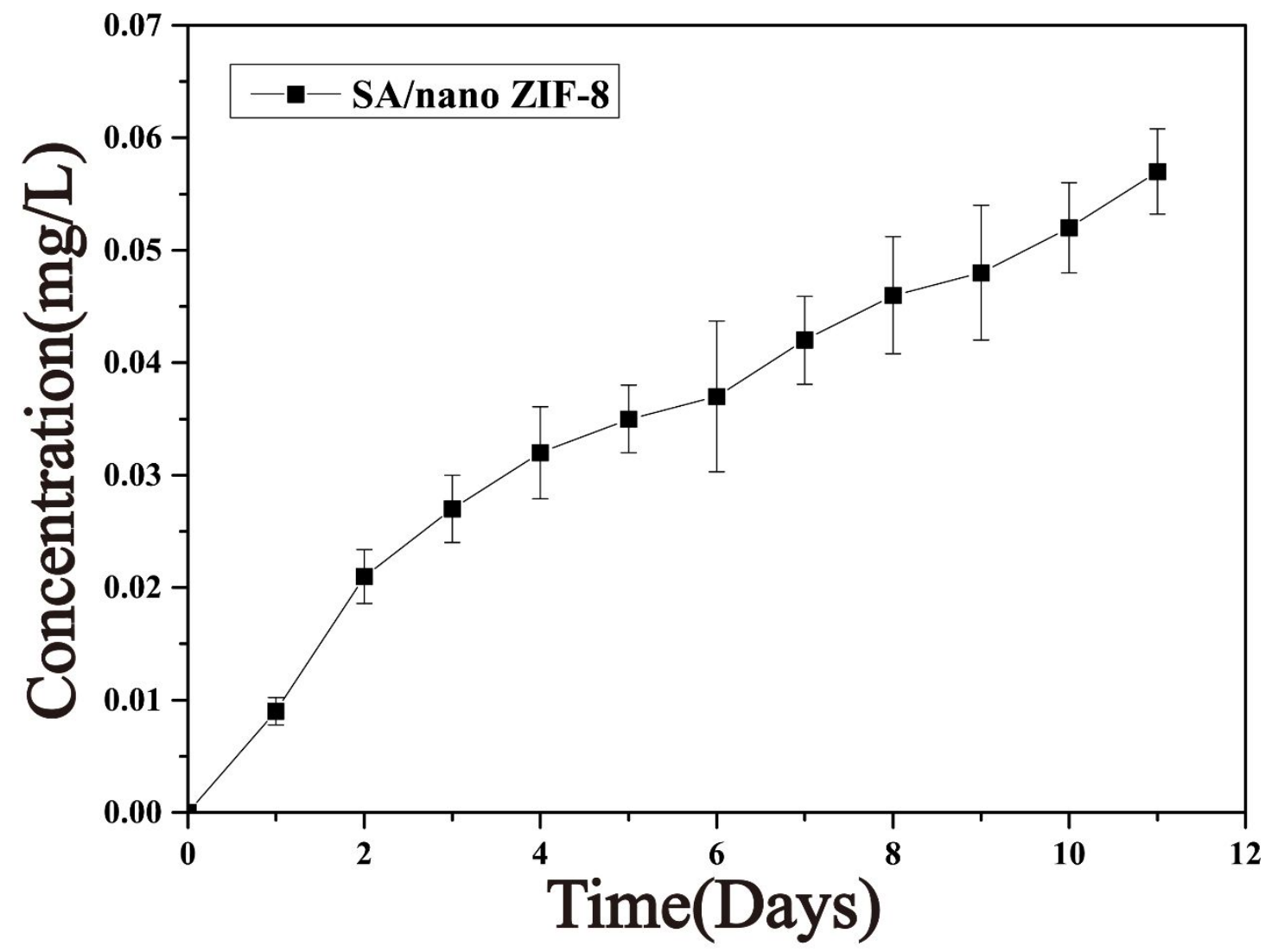

Figure S3. Zinc release from SA/nano ZIF-8 in PBS, according to ICP-MS. 


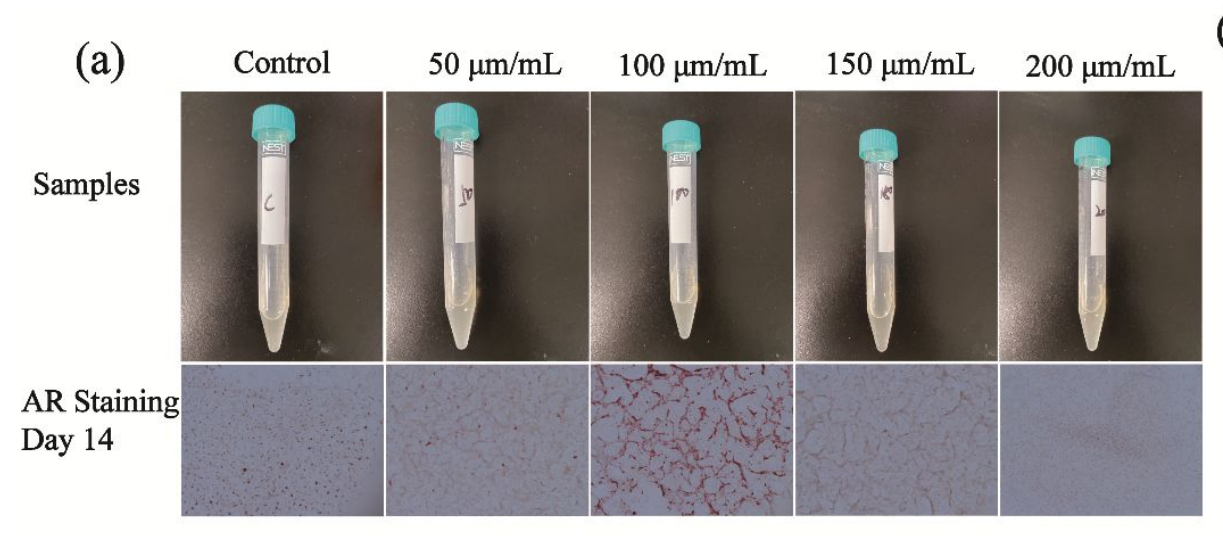

(b)
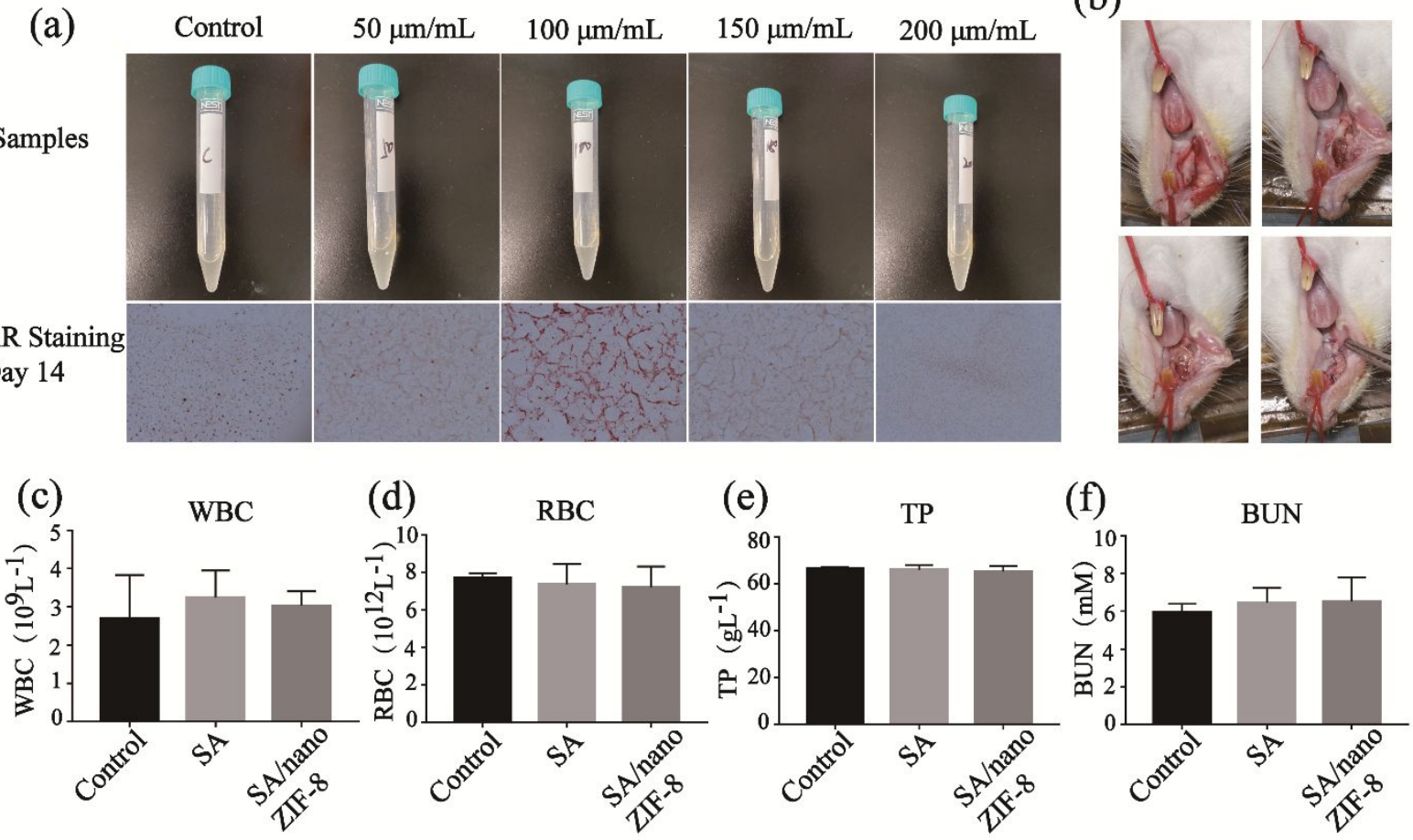

Figure S4. (a) AR staining of rBMSCs treated with nano ZIF-8 at different concentrations blended with SA after 14 day culturing. b) Brief illustration of critical-size rat alveolar defect model and surgical procedure. WBC (c), RBC (d), TP (e) and BUN (f) of the blood samples collected 4 weeks after the maxillary defect surgery and implantation. $n=3$. 


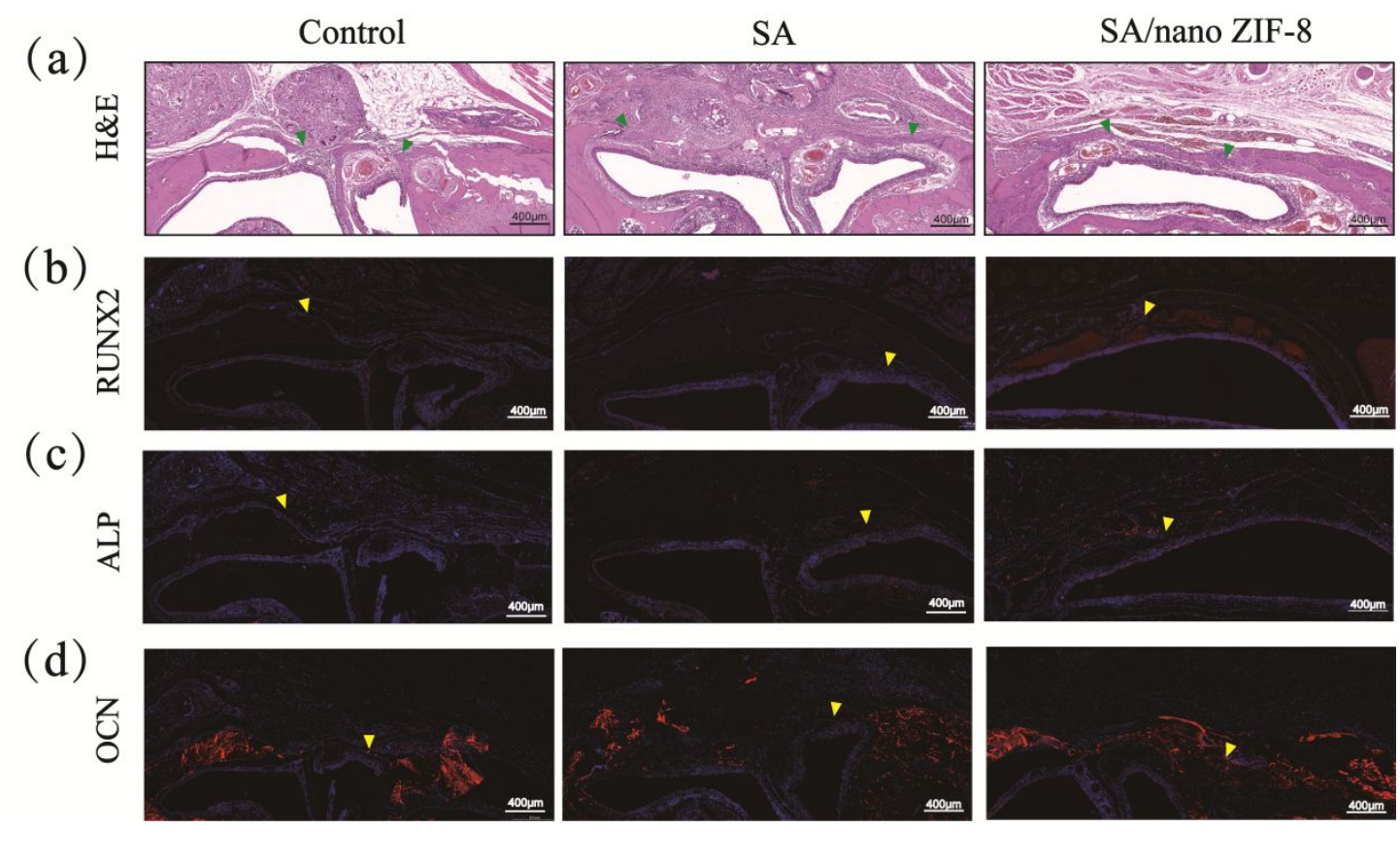

Figure S5. SA/nano ZIF-8 gel promoted bone regeneration of rat premaxillary critical-size defects by influencing the activity of rBMSCs. (a) H\&E staining of regenerated bone. (Green trangles show new bone.) RUNX2 (b), ALP (c) and OCN (d) immunofluorescence staining of bone defects. (Yellow triangles show the indicated markers expression.) 
(a)

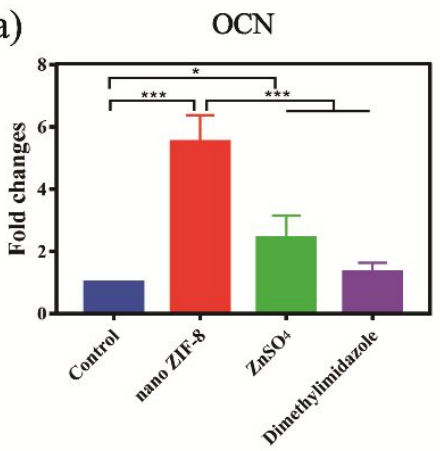

(b)

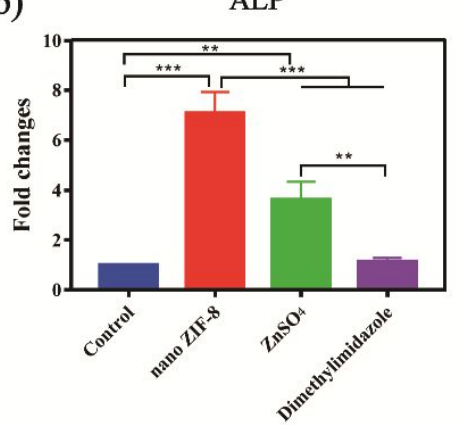

(c)

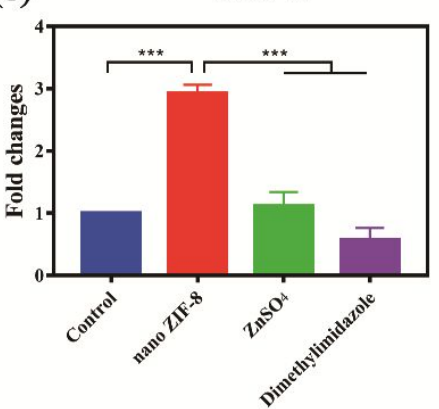

Figure S6. Quantitative analysis of OCN (a), ALP (b) and VEGF- $\alpha$ (c) secreted by four different materials induction after 14 days. All data were quantified using the Image $\mathrm{J}$ software. $n=3 .{ }^{*} \mathrm{P}<0.05,{ }^{*} * \mathrm{P}<0.01, * * * \mathrm{P}<0.001$ between each group. 
(a)

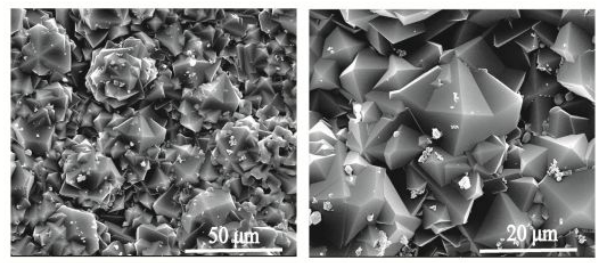

(c)



(d)

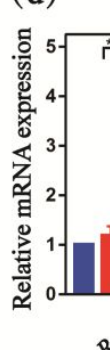

(b)

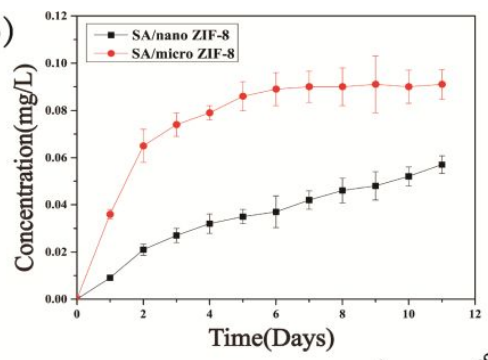

(e)



Figure S7. In vivo evaluation of the capacity of nano ZIF-8 and micro ZIF-8 to alter osteogenic differentiation. (a) SEM of synthesized micro ZIF-8. (b) Zinc release from SA/nano ZIF-8 and SA/ nano ZIF-8 in PBS, according to ICP-MS. PCR results at day 3 (c) and day 10 (d). (e) Western blot results after 10 days. $* \mathrm{P}<0.05$, $* * \mathrm{P}<0.01, * * * \mathrm{P}<0.001$ between each group. \#, \#\#, \#\# indicate significant differences in comparision with the control group. $\quad{ }^{\#} \mathrm{P}<0.05, \quad{ }^{\#} \mathrm{P}<0.01, \quad{ }^{\# \#} \mathrm{P}<0.001$. 
(a) nano ZIF-8 vs control (up): Top 30 GO Term

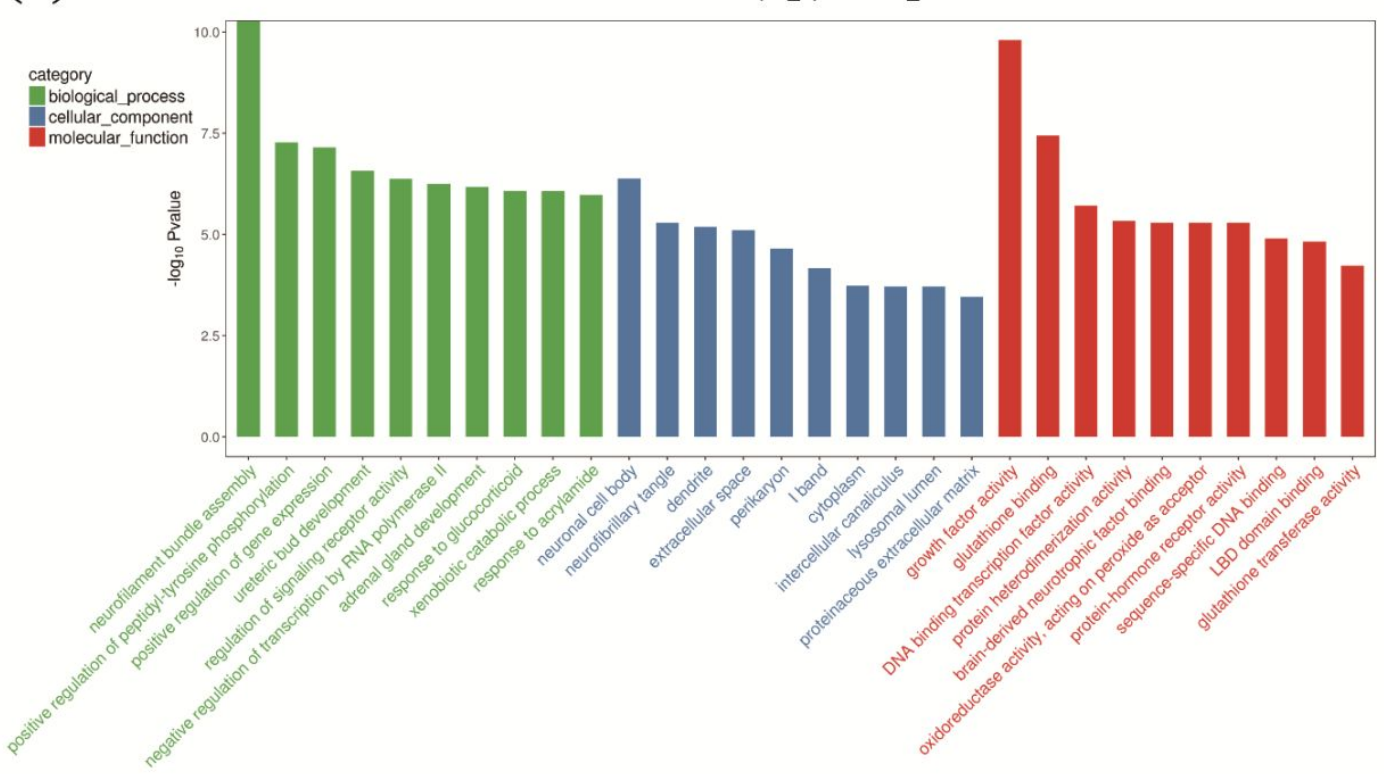

(b) nano ZIF-8 vs control (down): Top 30 GO Term

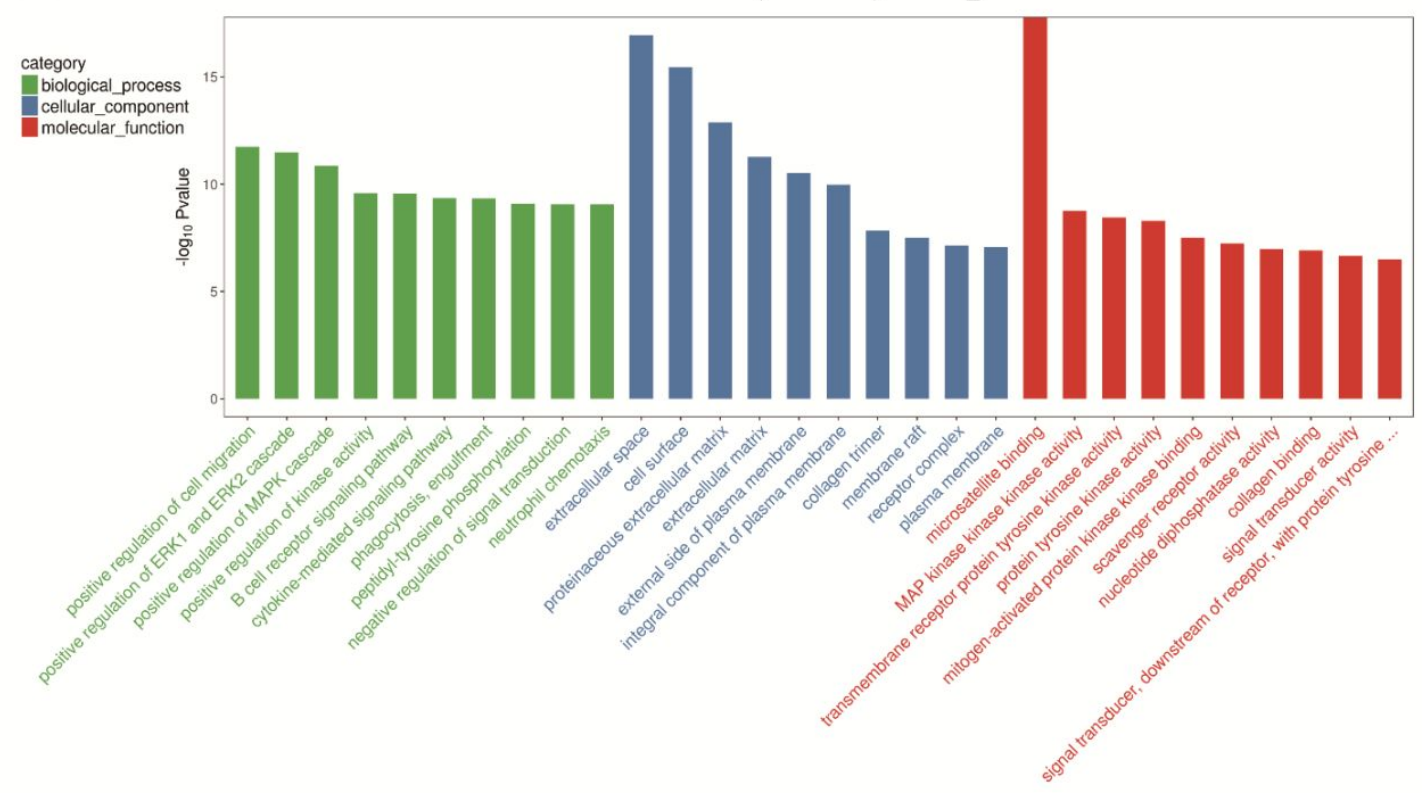

Figure S8. Top 30 upregulated (a) and downregulated (b) GO terms of rBMSCs after adding nano ZIF-8 for 4 days. 
(a)

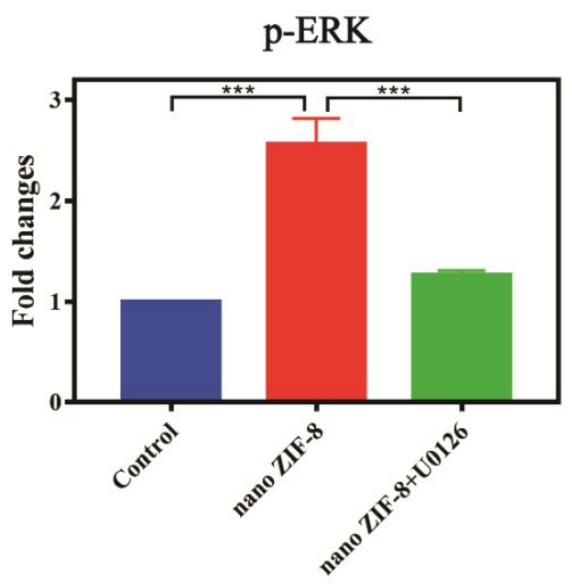

(c)

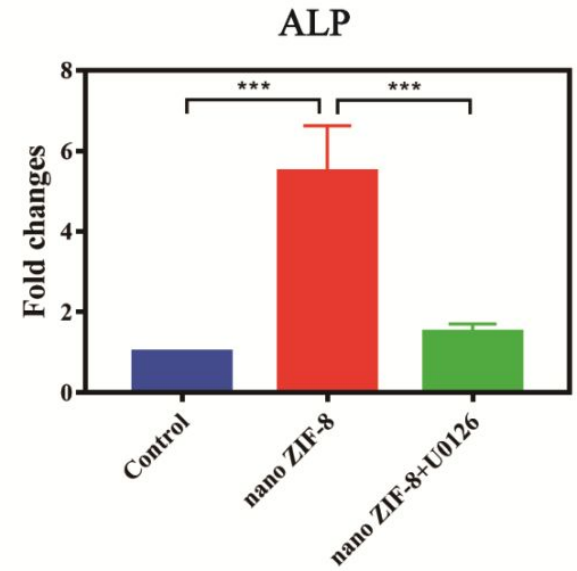

(b)

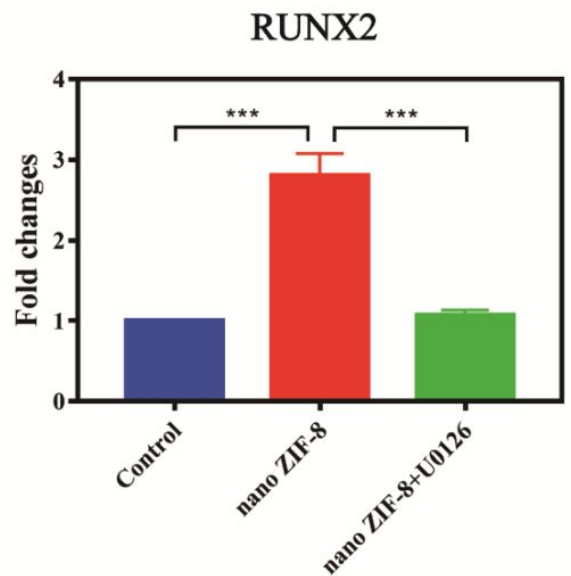

(d)

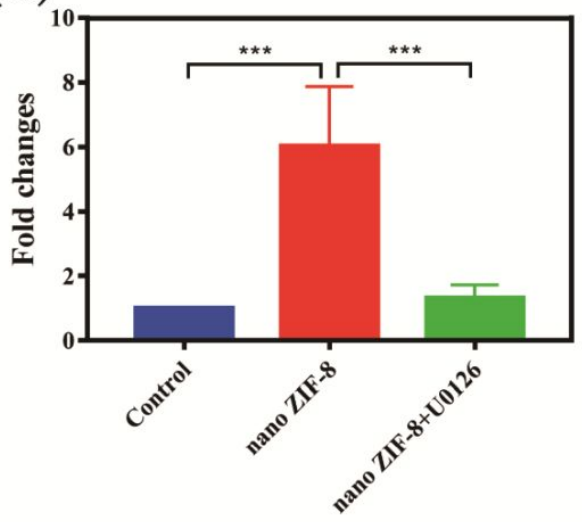

Figure S9. Quantitative analysis of pERK (a), RUNX2 (b) expression after 7 days and ALP (c), OCN (d) after 14 days. All data were quantified using the Image $\mathrm{J}$ software. $n=3$. ${ }^{*} \mathrm{P}<0.05, * * \mathrm{P}<0.01,{ }^{* * *} \mathrm{P}<0.001$ between each group. 


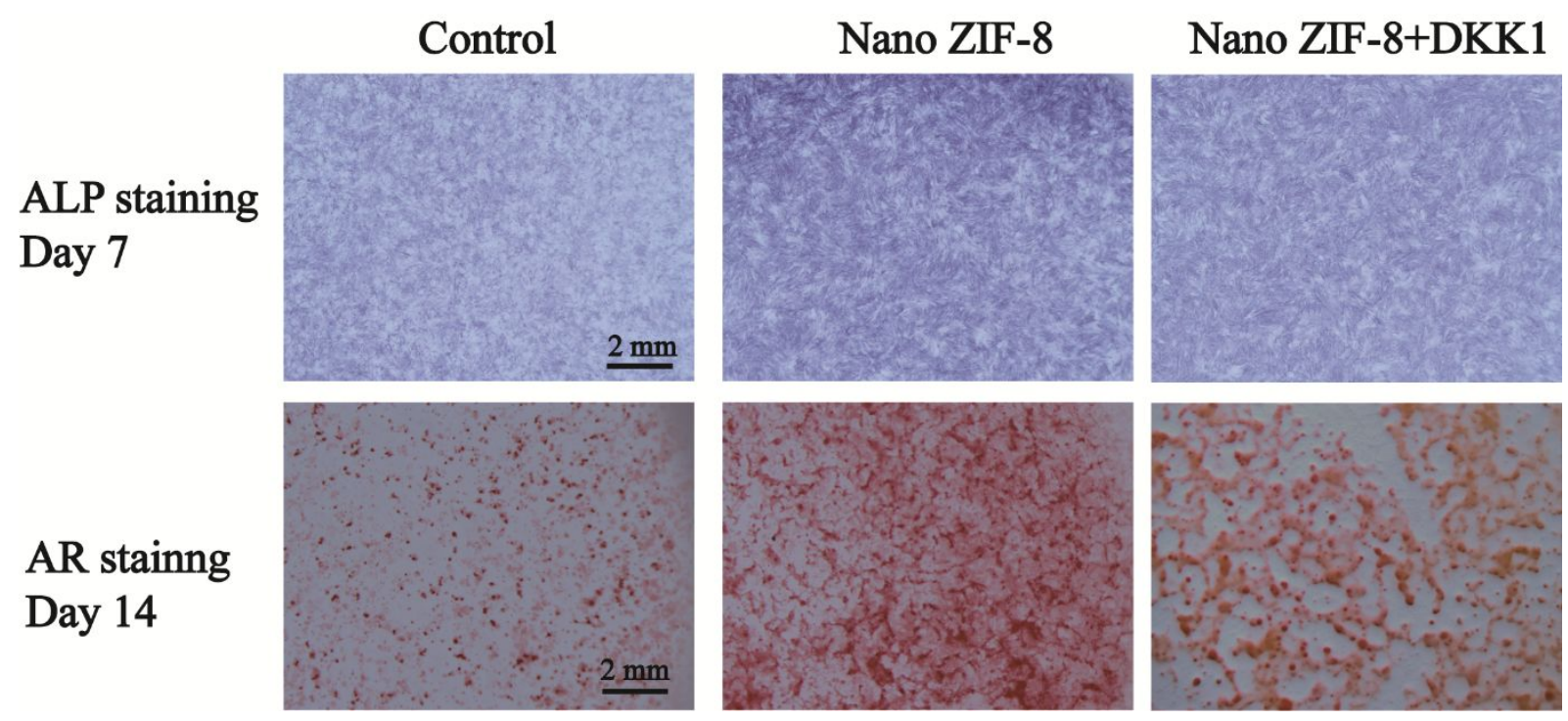

Figure S10. ALP staining and matrix mineralization of rBMSCs adding nano ZIF-8 and DKK-1. 
Table S1. Real time-PCR primer sequences.

\begin{tabular}{lll}
\hline Name & Primer & Sequence $\left(5^{\prime}-->3^{\prime}\right)$ \\
\hline Runx 2 & Forward & CACAAGTGCGGTGCAAACTT \\
Runx2 & Reverse & AAGAGGCTGTTTGACGCCAT \\
Alp & Forward & CCTGCCTGCAGGATCGGAA \\
Alp & Reverse & TTCTCTGGCACAAATGAGTTGGTA \\
Sppl(Opn) & Forward & ACACAGCGTCTCAACCTCAA \\
Sppl(Opn) & Reverse & AGAACCAAAGCACCCCTGTC \\
Bglap $(\mathrm{Ocn})$ & Forward & TTATTGTTTGAGGGGCCTGGG \\
Bglap $(\mathrm{Ocn})$ & Reverse & ACACAACTGCAGGTCGAGTTT \\
\hline
\end{tabular}

